\title{
Renal Teratoma: A Rare Pediatric Tumor
}

El Badri $\mathrm{Y}^{1^{*}}$, Gakosso $\mathrm{C}^{1}$, Basraoui $\mathrm{D}^{1}$, Jalal $\mathrm{H}^{1}$

${ }^{1}$ Radiology department, Mother and son hospital, Mohammed VI University Hospital, Cadi Ayyad University, Marrakech, Morocco

DOI: $\underline{10.36347 / \text { sjmcr.2021.v09i03.032 }}$

| Received: 02.03.2021 | Accepted: 19.03.2021 | Published: 30.03.2021

*Corresponding author: El Badri Y

\section{Abstract}

Intra renal teratoma is an extremely rare tumor. Only few cases have been published in the literature. In its renal location, the diagnosis of teratoma is rarely mentioned preoperatively since its clinical and radiological signs are not very specific, the final diagnosis is often confirmed by the pathological study. We report the case of a cystic intra renal teratoma in a three-month-old infant who underwent an enlarged nephrectomy with pathologic confirmation of the diagnosis. Surgery is the treatment of choice for intrarenal teratoma. It is usually not sensitive to chemotherapy, so this diagnosis should always be borne in mind in front of a cystic kidney mass in order to avoid chemotherapy that is harmful to these young patients.

Keywords: Teratoma, kidney, computed tomography (CT).

Copyright $($ C) 2021 The Author(s): This is an open-access article distributed under the terms of the Creative Commons Attribution 4.0 International License (CC BY-NC 4.0) which permits unrestricted use, distribution, and reproduction in any medium for non-commercial use provided the original author and source are credited.

\section{INTRODUCTION}

Teratoma is one of the germ cell tumors. It contains tissues different to the region that houses it, derived from embryonic multi- potential cells from three layers of the embryo. Their malignant potential is directly dependent on the degree of immaturity and the presence of neuroectoderm. The intrarenal location of teratomas is unusual. Few cases have been described in the literature [1], exceptionally mentioned preoperatively, because the clinical and radiological signs are not very specific. The definitive diagnosis is pathological.

We report a new pediatric case of intrarenal teratoma. On his admission, the clinical and radiological signs did not allow to distinguish it from other histological types more frequently observed in children, in particular Wilms tumor or nephroblastoma.

This work aims to highlight the contribution of imaging in making the diagnosis of this fairly rare pathological entity in children.

\section{Case Report}

We report the case of a 3-month-old infant who presented a progressive abdominal distension from birth, complicated by respiratory distress.

On clinical examination there were a significant abdominal distension and a firm mass occupying the entire abdomen. An ultrasound made showed a large solido-cystic mass containing calcifications, seeming to depend on the right kidney.

The abdomino-pelvic CT revealed a large retroperitoneal mass of the right hemi abdomen, well limited measuring $12 \times 10.5 \times 15 \mathrm{~cm}$ (AP, T, CC), with three components (cystic, fatty and calcic), discreetly enhanced after injection of contrast; This mass seems to depend on the right kidney, pushing back the ipsilateral diaphragmatic pillar, the inferior vena cava, the aorta and its visceral and parietal branches and its bifurcation, the hepatic pedicle, the duodeno-pancreatic block, the loops and the mesentery; and comes into contact with the renal pedicle suggesting a very probable renal teratoma (figure 1). 


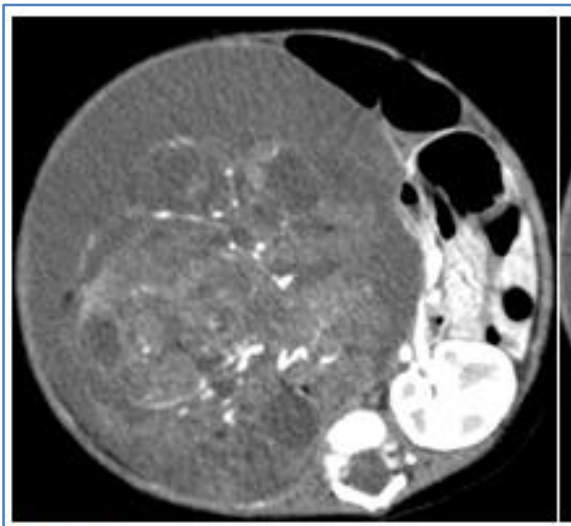

Fig 1- a

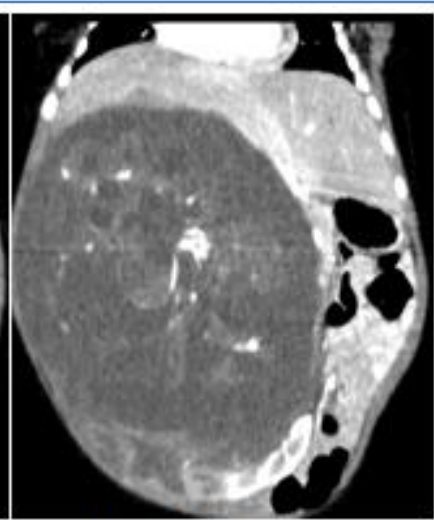

Fig 1-b

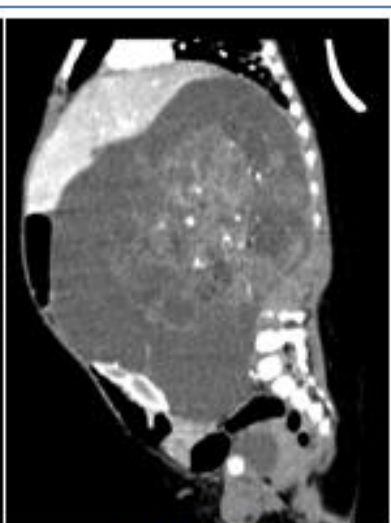

Fig 1 - c

Fig-1: Abdominal CT after injection of contrast axial section (a), coronal section (b) and sagittal section (c): Large retroperitoneal mass of the right flank, well-limited, with three components (cystic, fatty and calcium), discreetly enhanced after injection of contrast

The patient was operated (subumbilical laparotomy) and the exploration revealed a large retroperitoneal mass at the expense of the right kidney, with adhesions to the posterior abdominal wall and to the liver.

The operative procedure consisted of an enlarged right ureteronephectomy. The macroscopic appearance after its excision showed a solid mass with a cystic component. When opened, the lesion contained bone cartilage.

In our case, the pathological diagnosis retained was an intra-renal teratoma with minimal immature contingent. The operative consequences were simple. Regular clinical and paraclinical monitoring to detect recurrence has so far not been negative (12 months of follow-up), no suspicious lesions detected.

\section{DISCUSSION}

Teratoma is a malformative germ cell tumor derived from embryonic multipotential cells; it has a potential for multiple differentiation, which explains the heterogeneity of the tissues contained in the mass. They are most often benign; however, they are distinguished by their mature or immature character [2].

The intra-renal localization of teratomas is unusual. Murphy et al. [3] have proposed diagnostic criteria for intrarenal teratoma: the primary tumor must be intrarenal, delimited by the renal capsule, the absence of distant tumor may have given metastases in the kidney, and the renal tumor must show unequivocal heterologous organogenesis.

These different criteria were found in our observation. Renal teratoma manifests as an abdominal mass, abdominal pain, anorexia, vomiting, constipation, and hematuria. Sometimes they are accidentally detected [4].
Ultrasound reveals a cystic, solid or solidocystic mass, or hyperechoic with coarse foci of calcifications [5]. On standard radiograph, intra-renal teratomas may appear as a large unilateral abdominal masse with calcifications, the presence of formed bone components such as phalanges is pathognomonic of teratoma.

On CT, retroperitoneal teratoma may appear as a complex mass containing a well-circumscribed fluid component, fatty tissue, and calcifications [6]. The presence of fat in the cyst and calcifications in the cyst wall is very suggestive of a cystic teratoma.

In our patient, computed tomography revealed a large retroperitoneal lesion with a pathognomonic radiologic appearance of a teratoma.

Nevertheless, if a renal teratoma occurs during infancy, it can be confused with Wilms tumor, neuroblastoma or other intra-abdominal malignant tumors like carcinomas with fatty component or angiomyolipoma in the context of tuberous sclerosis of Bourneville [7].

Complete surgical resection is the treatment of choice, but this is not always possible due to the large size of the tumor or the proximity of vital structures [8].

\section{Conclunsion}

Although rare, infants' intrarenal teratoma should be considered in the list of differential diagnoses of pediatric intrarenal masses to avoid chemotherapy that might be harmful to young patients.

\section{REFERENCES}

1. García-Galvis OF, Stolnicu S, Muñoz E. Adultextrarenal Wilms tumor of the uteruswithteratoidfeatures. Huma pathol. 2009;40:418-424. 
2. MouadStatoua, AbdellatifKoutani. Tératome mature géantrétropéritonéale. Pan African Medical Journal. 2014; 18:147.

3. Murphy WM, Bruce Beckwith J, Farrow GM. Tumors ofthe kidney. In: Rosai J (ed) Tumors of the kidney, bladder, andrelated urinary structures. (Thirs Sciences, Fascicle 11). WashingtonDC, Armed Forces Institute of pathology. 1993; 90

4. 3.Alexander M, Alexander $\mathrm{K}$, Uta B, Holger T, Wolfgang H, Ivo L, Nicole S, Christian W, Thomas A. Mixed hepatoblastoma and teratoma of the liverin a 3-year-old child: a unique combination and clinical challenge. DiagnPathol. 2009; 4:37.
5. Jones NM, Kiely EM. Retroperitonealteratomaspotential for surgicalmisadventure. J PediatrSurg. 2008; 43:184-186.

6. Nirmal TJ, Krishnamoorthy S, Korula A. Primaryintrarenalteratoma in an adult: a case report and review of literature. Indian J Urol. 2009; 25:404-406.

7. Kyoko M, Yasuharu O, Yukiko T, Takashi K, Masahiko O, Akira K, Hiroyuki M, Mitsuru N, Tomayoshi $\mathrm{H}$. Congenitalintrarenalteratomaarisingfromahorsesho ekidney. Jpedsurg. 2006;03 019.

8. Han Chu1, Qi-Fei Deng1, Xiang Liu, Bo Peng, Yong-Sheng Cao. Kidney teratoma: A case report and literature review.j.eucr.2018.07.012. 